\title{
Numerical stability and oscillation of the Runge-Kutta methods for the differential equations with piecewise continuous arguments alternately of retarded and advanced type
}

Minghui Song* and Mingzhu Liu

"Correspondence: songmh@lsec.cc.ac.cn

Department of Mathematics, Harbin Institute of Technology, Harbin,

150001, P.R. China

\begin{abstract}
This paper is concerned with the numerical properties of Runge-Kutta methods for the alternately of retarded and advanced equation $\dot{x}(t)=a x(t)+a_{0} x\left(2\left[\frac{t+1}{2}\right]\right)$. The stability region of Runge-Kutta methods is determined. The conditions that the analytic stability region is contained in the numerical stability region are obtained. A necessary and sufficient condition for the oscillation of the numerical solution is given. And it is proved that the Runge-Kutta methods preserve the oscillations of the analytic solutions. Some numerical experiments are illustrated.
\end{abstract}

Keywords: stability; oscillation; delay differential equation; piecewise continuous arguments

\section{Introduction}

This paper deals with the numerical solution of the alternately of retarded and advanced equation with piecewise continuous arguments (EPCA)

$$
\dot{x}(t)=f\left(x(t), x\left(2\left[\frac{t+1}{2}\right]\right)\right)
$$

where [.] is the greatest integer function. Differential equations of this form have stimulated considerable interest and have been studied by Cooker and Wiener [1], Jayasree and Deo [2], Wiener and Aftabizadeh [3]. In these equations the argument deviation $T(t)=t-2\left[\frac{t+1}{2}\right]$ is a piecewise linear periodic function with periodic 2. Also, $T(t)$ is negative for $2 n-1 \leq t<2 n$ and positive for $2 n \leq t<2 n+1$. Therefore, (1.1) is of advanced type on $[2 n-1,2 n)$ and of retarded type on $(2 n, 2 n+1)$.

EPCA describe hybrid dynamical systems, combine properties of both differential and difference equations and have applications in certain biomedical models in the work of Busenberg and Cooke [4]. For these equations of mixed type, the change of sign in the argument deviation leads not only to interesting periodic properties, but also to complications in the asymptotic and oscillatory behavior of solutions. Oscillatory, stability and 
periodic properties of the linear EPCA alternately of retarded and advanced form have been investigated in [1].

There are some papers concerning the stability of numerical solutions of delay differential equations with piecewise continuous arguments, such as [5-7]. Also, there have been results concerning oscillations of delay differential equations and delay difference equations, even including delay differential equations with piecewise continuous arguments [8]. But there is no paper concerned with the stability and oscillation of the numerical solutions of Eq. (1.1).

In this paper, we investigate the numerical properties, including the stability and oscillation, of Runge-Kutta methods of delay differential equations with piecewise continuous arguments.

We consider the following equation:

$$
\left\{\begin{array}{l}
\dot{x}(t)=a x(t)+a_{0} x\left(2\left[\frac{t+1}{2}\right]\right) \\
x(0)=x_{0}
\end{array}\right.
$$

where $a, a_{0}$ are constants and [.] is the greatest integer function.

Definition 1.1 [9] A solution of $(1.2)$ on $[0, \infty)$ is a function $x(t)$ that satisfies the conditions:

1. $x(t)$ is continuous on $[0, \infty)$.

2. The derivative $\dot{x}(t)$ exists at each point $t \in[0, \infty)$, with the possible exception of the points $t=2 n-1$ for $n \in \mathbb{N}$, where one-sided derivatives exist.

3. (1.2) is satisfied on each interval $[2 n-1,2 n+1)$ for $n \in \mathbb{N}$.

In the following, we use these notations

$$
m(t)=e^{a t}+\left(e^{a t}-1\right) a^{-1} a_{0}, \quad m_{1}=m(1), \quad m_{-1}=m(-1) .
$$

The following theorems give existence and uniqueness of solutions and provide necessary and sufficient conditions for the asymptotic stability and the oscillation of all solutions of (1.2).

Theorem 1.2 [9] Assume that $a, a_{0}, x_{0} \in \mathbb{R}$. Then the initial value problem (1.2) has on $[0, \infty)$ a unique solution $x(t)$ given by

$$
x(t)=m(T(t))\left(\frac{m_{1}}{m_{-1}}\right)^{\left[\frac{t+1}{2}\right]} x_{0}
$$

where $T(t)=t-2\left[\frac{t+1}{2}\right], x(0)=x_{0}$.

Theorem 1.3 [9] The solution $x(t)=0$ of $(1.2)$ is asymptotically stable $\left(\lim _{t \rightarrow \infty} x(t)=0\right)$ if and only if any one of the following hypotheses is satisfied:

1. $a<0, a_{0}>-\frac{a\left(e^{2 a}+1\right)}{\left(e^{a}-1\right)^{2}}$ or $a_{0}<-a$;

2. $a>0,-\frac{a\left(e^{2 a}+1\right)}{\left(e^{a}-1\right)^{2}}<a_{0}<-a$;

3. $a=0, a_{0}<0$. 
In the following, we give the definition of oscillation and non-oscillation.

Definition 1.4 A nontrivial solution of (1.2) is said to be oscillatory if there exists a sequence $\left\{t_{k}\right\}_{k=1}^{\infty}$ such that $t_{k} \rightarrow \infty$ as $k \rightarrow \infty$ and $x\left(t_{k}\right) x\left(t_{k-1}\right) \leq 0$. Otherwise, it is called non-oscillatory. We say (1.2) is oscillatory if all nontrivial solutions of (1.2) are oscillatory. We say (1.2) is non-oscillatory if all nontrivial solutions of (1.2) are non-oscillatory.

Consider the difference equation

$$
a_{n+k}+p_{1} a_{n+k-1}+\cdots+p_{k} a_{n}=0, \quad n=0,1,2, \ldots,
$$

where $k=1,2, \ldots, p_{i} \in \mathbb{R}, i=1,2, \ldots, k$, and its associated characteristic equation is

$$
\lambda^{k}+p_{1} \lambda^{k-1}+\cdots+p_{k-1} \lambda+p_{k}=0 .
$$

Definition 1.5 A nontrivial solution $\left\{a_{n}\right\}$ of (1.3) is said to be oscillatory if there exists a sequence $\left\{n_{k}\right\}$ such that $n_{k} \rightarrow \infty$ as $k \rightarrow \infty$ and $a_{n_{k}} a_{n_{k}-1} \leq 0$. Otherwise, it is called nonoscillatory. Equation (1.3) is said to be oscillatory if all nontrivial solutions of Eq. (1.3) are oscillatory. Equation (1.3) is called non-oscillatory if all nontrivial solutions of Eq. (1.3) are non-oscillatory.

Theorem 1.6 [10] Eq. (1.3) is oscillatory if and only if the characteristic equation (1.4) has no positive roots.

Theorem 1.7 [9] A necessary and sufficient condition for all solutions of Eq. (1.2) to be oscillatory is either $a_{0}<-\frac{a e^{a}}{e^{a}-1}$ or $a_{0}>\frac{a}{e^{a}-1}$.

\section{Runge-Kutta methods}

In this section we consider the adaptation of the Runge-Kutta methods $(A, b, c)$. Let $h=\frac{1}{m}$ be a given step-size with an integer $m \geq 1$, and let the grid-points $t_{n}$ be defined by $t_{n}=n h$ $(n=0,1,2, \ldots)$.

For the Runge-Kutta methods, we always assume that $b_{1}+b_{2}+\cdots+b_{v}=1$ and $0 \leq c_{1} \leq$ $c_{2} \leq \cdots \leq c_{v} \leq 1$.

The adaptation of the Runge-Kutta methods to (1.2) leads to a numerical process of the following type:

$$
\left\{\begin{array}{l}
x_{n+1}=x_{n}+h \sum_{i=1}^{v} b_{i}\left(a y_{i}^{(n)}+a_{0} z_{i}^{(n)}\right) \\
y_{i}^{(n)}=x_{n}+h \sum_{j=1}^{v} a_{i j}\left(a y_{j}^{(n)}+a_{0} z_{j}^{(n)}\right)
\end{array}\right.
$$

where the matrix $A=\left(a_{i j}\right)_{v \times v}$, vectors $b=\left(b_{1}, b_{2}, \ldots, b_{v}\right)^{T}, c=\left(c_{1}, c_{2}, \ldots, c_{v}\right)^{T}$, and $x_{n}$ is an approximation to $x(t)$ at $t_{n}(n=1,2,3, \ldots) \cdot y_{i}^{(n)}$ and $z_{i}^{(n)}$ are approximations to $x\left(t_{n}+c_{i} h\right)$ and $x\left(2\left[\frac{t_{n}+c_{i} h}{2}\right]\right)$ respectively. Let $n=2 k m+l, l=-m,-m+1, \ldots, m-1$ for $k \geq 1, l=0,1, \ldots, m-1$ for $k=0$. Then $z_{i}^{(2 k m+l)}$ can be defined as $x_{2 k m}$ according to Definition $1.1(i=1, \ldots, v)$.

Let $Y^{(n)}=\left(y_{1}^{(n)}, y_{2}^{(n)}, \ldots, y_{v}^{(n)}\right)^{T}$. Then $(2.1)$ reduces to

$$
\left\{\begin{array}{l}
x_{2 k m+l+1}=x_{2 k m+l}+h a b^{T} Y^{2 k m+l}+h a_{0} x_{2 k m} \\
Y^{2 k m+l}=x_{2 k m+l} e+h a A Y^{2 k m+l}+h a_{0} A e x_{2 k m}
\end{array}\right.
$$


where $e=(1,1, \ldots, 1)^{T}$. Hence we have

$$
x_{2 k m+l+1}=R(x) x_{2 k m+l}+\frac{a_{0}}{a}(R(x)-1) x_{2 k m},
$$

where $x=h a, R(x)=1+x b^{T}(I-x A)^{-1} e$ is the stability function of the method.

We can obtain from (2.3)

$$
\begin{aligned}
& x_{2 k m+l}=\left(R^{l}(x)+\frac{a_{0}}{a}\left(R^{l}(x)-1\right)\right) x_{2 k m}, \\
& x_{2(k+1) m}=\frac{R^{m}(x)+\frac{a_{0}}{a}\left(R^{m}(x)-1\right)}{R^{-m}(x)+\frac{a_{0}}{a}\left(R^{-m}(x)-1\right)} x_{2 k m}, \\
& x_{2(k+1) m}=\frac{1+a_{0}}{1-a_{0}} x_{2 k m}, \quad a=0 .
\end{aligned}
$$

\section{Stability and oscillation of the Runge-Kutta methods}

In this section we discuss stability and oscillation of the Runge-Kutta methods.

\subsection{Numerical stability}

Definition 3.1 The Runge-Kutta method is called asymptotically stable at $\left(a, a_{0}\right)$ if there exists a constant $M$ such that $x_{n}$ defined by (2.1) tends to zero as $n \rightarrow \infty$ for all $h=\frac{1}{m}$ $(m>M)$ and any given $x_{0}$.

Definition 3.2 The set of all points $\left(a, a_{0}\right)$ at which the Runge-Kutta method is asymptotically stable is called an asymptotic stability region denoted by $S$.

For any given Runge-Kutta method, $R(x)=\frac{P(x)}{Q(x)}$, where $P(x)$ and $Q(x)$ are polynomials. $R(x)$ is a continuous function at the neighborhood of zero, and $R(0)=R^{\prime}(0)=1$. So there are $\delta_{1}<0<\delta_{2}$ such that

$$
\left\{\begin{array}{l}
1<R(x)<\infty \quad \text { for } 0<x<\delta_{2} \\
0<R(x)<1 \quad \text { for } \delta_{1}<x<0
\end{array}\right.
$$

which implies

$$
0<\frac{R(x)-1}{x}<\infty \quad \text { for } \delta_{1}<x<\delta_{2}
$$

Remark 3.3 It is known from [11] that $R(x)$ is an increasing function in $[-1,1]$, and $1<$ $R(x)<\infty$ for $0<x \leq 1,0<R(x)<1$ for $-1 \leq x<0$. Hence we can take $\delta_{1}=-1, \delta_{2}=1$ for simplicity.

In the following, we always suppose $h<\frac{1}{|a|}$.

It is easy to see from (2.4) and (2.5) that $x_{n} \rightarrow 0$ as $n \rightarrow \infty$ if and only if $x_{2 k m} \rightarrow 0$ as $k \rightarrow \infty$. Hence we have the following theorem.

Theorem 3.4 The Runge-Kutta method is asymptotically stable if any one of the following hypotheses is satisfied:

(i) $-\frac{a\left(R^{2 m}(x)+1\right)}{\left(R^{m}(x)-1\right)^{2}}<a_{0}<-a, a>0$; 
(ii) $-\frac{a\left(R^{2 m}(x)+1\right)}{\left(R^{m}(x)-1\right)^{2}}<a_{0}$ or $a_{0}<-a, a<0$;

(iii) $a_{0}<0, a=0$.

Proof In view of (2.5), the Runge-Kutta method is asymptotically stable if and only if

$$
\begin{aligned}
& -1<\frac{\left(a+a_{0}\right) R^{2 m}(x)-a_{0} R^{m}(x)}{\left(a+a_{0}\right)-a_{0} R^{m}(x)}<1, \quad a \neq 0, \\
& a_{0}<0, \quad a=0 .
\end{aligned}
$$

If $\left(a+a_{0}\right)-a_{0} R^{m}(x)>0$, then (3.3) reduces to

$$
a_{0}>-\frac{a\left(R^{2 m}(x)+1\right)}{\left(R^{m}(x)-1\right)^{2}} \quad \text { and } \quad\left(a+a_{0}\right) R^{2 m}(x)<a+a_{0}
$$

which is equivalent to

$$
\begin{aligned}
& a_{0}>-\frac{a\left(R^{2 m}(x)+1\right)}{\left(R^{m}(x)-1\right)^{2}}, \quad a \leq 0, \\
& -\frac{a\left(R^{2 m}(x)+1\right)}{\left(R^{m}(x)-1\right)^{2}}<a_{0}<-a, \quad a>0 .
\end{aligned}
$$

If $a+a_{0}-a_{0} R^{m}(x)<0$, then (3.3) reduces to

$$
a_{0}<-\frac{a\left(R^{2 m}(x)+1\right)}{\left(R^{m}(x)-1\right)^{2}} \quad \text { and } \quad\left(a+a_{0}\right) R^{2 m}(x)>a+a_{0}
$$

which is equivalent to

$$
a_{0}<-a, \quad a<0 .
$$

By virtue of (3.4) and (3.5), the theorem is proved.

\subsection{Numerical oscillations}

Theorem 3.5 The following statements are equivalent:

1. $\left\{x_{n}\right\}$ is oscillatory;

2. $\left\{x_{2 \mathrm{~km}}\right\}$ is oscillatory;

3. $a_{0}<\frac{-a R^{m}(x)}{R^{m}(x)-1}$, or $a_{0}>\frac{a}{R^{m}(x)-1}$.

Proof $\left\{x_{2 k m}\right\}$ is not oscillatory if and only if

$$
\frac{R^{m}(x)+\frac{a_{0}}{a}\left(R^{m}(x)-1\right)}{R^{-m}(x)+\frac{a_{0}}{a}\left(R^{-m}(x)-1\right)}>0
$$

i.e.,

$$
\frac{-a R^{m}(x)}{R^{m}(x)-1}<a_{0}<\frac{a}{R^{m}(x)-1} .
$$


Hence for any $l=1,2, \ldots, m-1$

$$
\frac{-a R^{l}(x)}{R^{l}(x)-1}<\frac{-a R^{m}(x)}{R^{m}(x)-1}<a_{0}<\frac{a}{R^{m}(x)-1}<\frac{a}{R^{l}(x)-1},
$$

which is equivalent to

$$
\begin{aligned}
& R^{l}(x)+\frac{a_{0}}{a}\left(R^{l}(x)-1\right)>0, \\
& R^{-l}(x)+\frac{a_{0}}{a}\left(R^{-l}(x)-1\right)>0 .
\end{aligned}
$$

We obtain from (2.4) that $\left\{x_{n}\right\}$ is not oscillatory.

Moreover, $\left\{x_{2 \mathrm{~km}}\right\}$ is oscillatory if and only if

$$
\frac{R^{m}(x)+\frac{a_{0}}{a}\left(R^{m}(x)-1\right)}{R^{-m}(x)+\frac{a_{0}}{a}\left(R^{-m}(x)-1\right)}<0
$$

which is equivalent to

$$
a_{0}<\frac{-a R^{m}(x)}{R^{m}(x)-1}, \quad \text { or } \quad a_{0}>\frac{a}{R^{m}(x)-1} .
$$

\section{Preservation of stability and oscillations}

In this section, we investigate the conditions under which the analytic stability region is contained in the numerical stability region and the conditions under which the numerical solution and the analytic solution are oscillatory simultaneously. We also study the stability and oscillation of the Runge-Kutta method with the stability function which is given by the $(r, s)$-Padé approximation to $e^{z}$.

In order to do this, the following lemmas and corollaries will be useful to determine conditions.

Lemma 4.1 $[12,13]$ The $(r, s)$-Padé approximation to $e^{z}$ is given by

$$
R(z)=\frac{P_{r}(z)}{Q_{s}(z)}
$$

where

$$
\begin{aligned}
& P_{r}(z)=1+\frac{r}{r+s} z+\frac{r(r-1)}{(r+s)(r+s-1)} \frac{z^{2}}{2 !}+\cdots+\frac{r ! s !}{(r+s) !} \frac{z^{r}}{r !}, \\
& Q_{s}(z)=1-\frac{r}{r+s} z+\frac{s(s-1)}{(r+s)(r+s-1)} \frac{z^{2}}{2 !}-\cdots+(-1)^{s} \frac{s ! r !}{(r+s) !} \frac{z^{s}}{s !},
\end{aligned}
$$

with error

$$
e^{z}-R(z)=(-1)^{s} \frac{s ! r !}{(r+s) !(r+s+1) !} z^{r+s+1}+O\left(z^{r+s+2}\right) .
$$

It is the unique rational approximation to $e^{z}$ of order $r+s$, such that the degree of a numerator and a denominator are $r$ and s respectively. 
Lemma 4.2 $[12,13]$ If $R(z)$ is the $(r, s)$-Padé approximation to $e^{z}$, then

(i) there are $s$ bounded star sectors in the right-half plane, each containing a pole of $R(z)$

(ii) there are $r$ bounded white sectors in the left-half plane, each containing a zero of $R(z)$;

(iii) all sectors are symmetric with respect to the real axis.

Corollary $4.3[5,7]$ Suppose $R(z)$ is the $(r, s)$-Padé approximation to $e^{z}$. Then

1. $x>0$

$$
\begin{aligned}
& R(x)<e^{x} \text { for all } x>0 \text { if and only if s is even. } \\
& R(x)>e^{x} \text { for all } 0<x<\xi \text { if and only if } s \text { is odd. }
\end{aligned}
$$

2. $x<0$

$$
\begin{aligned}
& R(x)>e^{x} \text { for all } x<0 \text { if and only if } r \text { is even. } \\
& R(x)<e^{x} \text { for all } \eta<x<0 \text { if and only if } r \text { is odd. }
\end{aligned}
$$

Where $\xi>1$ is a real zero of $Q_{s}(z)$ and $\eta<-1$ is a real zero of $P_{r}(z)$.

\subsection{Preservation of stability}

We introduce the set $H$ consisting of all pairs $\left(a, a_{0}\right) \in \mathbb{R}^{2}$ at which the Runge-Kutta method is asymptotically stable. In the following we investigate the conditions which lead to $H \subseteq S$. For convenience, we divide the region $H$ into three parts

$$
\begin{aligned}
& H_{0}=\left\{\left(a, a_{0}\right) \in H: a=0\right\}, \\
& H_{1}=\left\{\left(a, a_{0}\right) \in H \backslash H_{0}: a<0\right\}, \\
& H_{2}=\left\{\left(a, a_{0}\right) \in H \backslash H_{0}: a>0\right\},
\end{aligned}
$$

and in the similar way we denote

$$
\begin{aligned}
& S_{0}=\left\{\left(a, a_{0}\right) \in S: a=0\right\}, \\
& S_{1}=\left\{\left(a, a_{0}\right) \in S \backslash S_{0}: a<0\right\}, \\
& S_{2}=\left\{\left(a, a_{0}\right) \in S \backslash S_{0}: a>0\right\} .
\end{aligned}
$$

It is easy to see that $H=H_{0} \cup H_{1} \cup H_{2}, S=S_{0} \cup S_{1} \cup S_{2}$ and

$$
H_{i} \cap H_{j}=\varnothing, \quad S_{i} \cap S_{j}=\varnothing, \quad H_{i} \cap S_{j}=\varnothing, \quad i \neq j, i, j=0,1,2 .
$$

Therefore, we can conclude that $H \subseteq S$ is equivalent to $H_{i} \subseteq S_{i}, i=0,1,2$.

Theorem 4.4 Suppose that the stability function $R(x)$ of the Runge-Kutta method is given by the $(r, s)$-Pade approximation to $e^{x}$. Then $H_{1} \subseteq S_{1}$ if and only if $r$ is odd and $H_{2} \subseteq S_{2}$ if and only if s is even.

Proof In view of Theorem 1.3 and Theorem 3.4, we have that $H \subseteq S$ if and only if

$$
-\frac{a\left(R^{2 m}(x)+1\right)}{\left(R^{m}(x)-1\right)^{2}} \leq-\frac{a\left(e^{2 a}+1\right)}{\left(e^{a}-1\right)^{2}}
$$


which is equivalent to

$$
R(x) \leq e^{x}
$$

since $f(x)=\frac{x^{2}+1}{(x-1)^{2}}$ is increasing in $[0,1)$ and decreasing in $(1, \infty)$.

According to Corollary 4.3, the proof is complete.

Theorem 4.5 For all Runge-Kutta methods, we have $H_{0}=S_{0}$.

Corollary 4.6 For the A-stable higher order Runge-Kutta methods, it is easy to see from Theorem 4.4 that

1. For the $v$-stage Radau IA and IIA methods, $H \subseteq S$ if and only if $v$ is even;

2. For the v-stage Lobatto IIIA and IIIB methods, $H_{1} \subseteq S_{1}$ if and only if $v$ is even and $\mathrm{H}_{2} \subseteq S_{2}$ if and only if $v$ is odd;

3. For the v-stage Gauss-Legerdre and Lobatto IIIC methods, $H_{1} \subseteq S_{1}$ if and only if $v$ is odd and $\mathrm{H}_{2} \subseteq S_{2}$ if and only if $v$ is even.

It is known that all $v$-stage explicit Runge-Kutta methods with $p=v=1,2,3,4$ possess the stability function (see [12])

$$
R(x)=1+x+\frac{x^{2}}{2 !}+\cdots+\frac{x^{p}}{p !},
$$

which is the $(v, 0)$-Padé approximation to $e^{x}$.

Theorem 4.7 For the v-stage explicit Runge-Kutta methods with $p=v=1,2,3,4, H \subseteq S$ if and only if $v$ is odd.

\subsection{Preservation of oscillations}

Definition 4.8 We call that the Runge-Kutta methods preserve oscillations of Eq. (1.2) if (1.2) oscillates, which implies that there is an $h_{0}$ such that (2.4) oscillates for $h<h_{0}$.

Owing to Theorem 1.7 and Theorem 3.5, the Runge-Kutta method preserves the oscillation of (1.2) if and only if

$$
\frac{-a e^{a}}{e^{a}-1} \leq \frac{-a R^{m}(x)}{R^{m}(x)-1} \quad \text { or } \quad \frac{a}{e^{a}-1}>\frac{a}{R^{m}(x)-1} .
$$

Theorem 4.9 Suppose that the stability function $R(x)$ is given by the $(r, s)$-Pade approximation to $e^{x}$, then the Runge-Kutta method preserves the oscillation of (1.2) if and only if

$$
R(x) \geq e^{x} \quad \text { for } a>0
$$

and

$$
R(x) \leq e^{x} \quad \text { for } a<0 .
$$




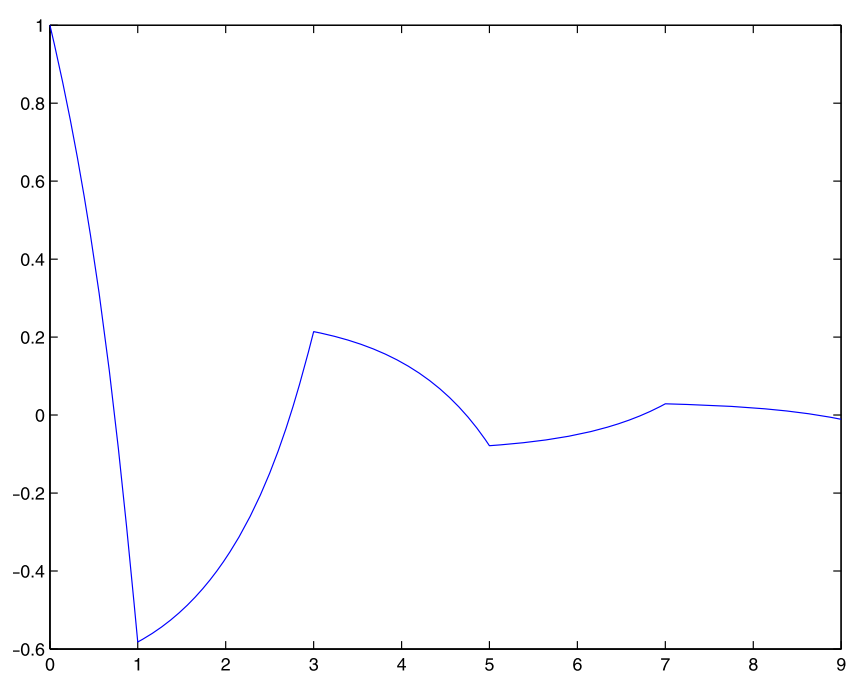

Figure 1 2-Gauss method for (5.1).

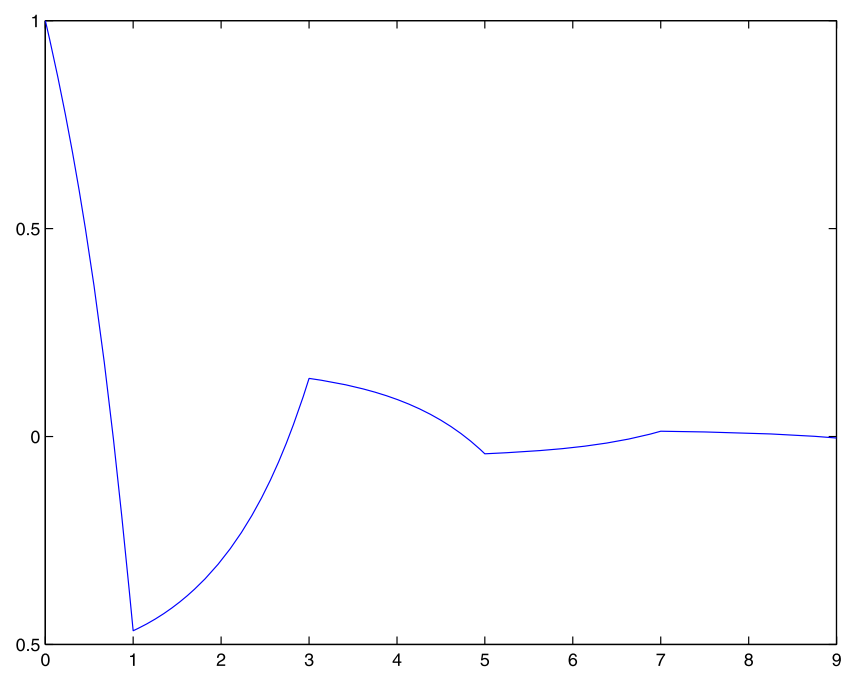

Figure 2 Explicit Euler method for (5.1).

Proof The proof is completed by $(4.3)$ and noting that $\frac{x}{x-1}$ is decreasing in $[0,1)$.

\section{Corollary 4.10}

1. The v-stage Gauss-Legendre and Lobatto IIIC methods preserve the oscillation of Eq. (1.2) if and only if $v$ is odd.

2. The v-stage Lobatto IIIA and IIIB methods preserve the oscillation of Eq. (1.2) if and only if $v$ is even.

3. The $v$-stage Radau IA and IIA methods preserve the oscillation of Eq. (1.2) if $v$ is odd for $a>0$ and if $v$ is even for $a<0$.

Theorem 4.11 The v-stage explicit Runge-Kutta methods with $p=v=1,2,3,4$ preserve the oscillation of Eq. (1.2) with $a<0$ if $v$ is odd. 


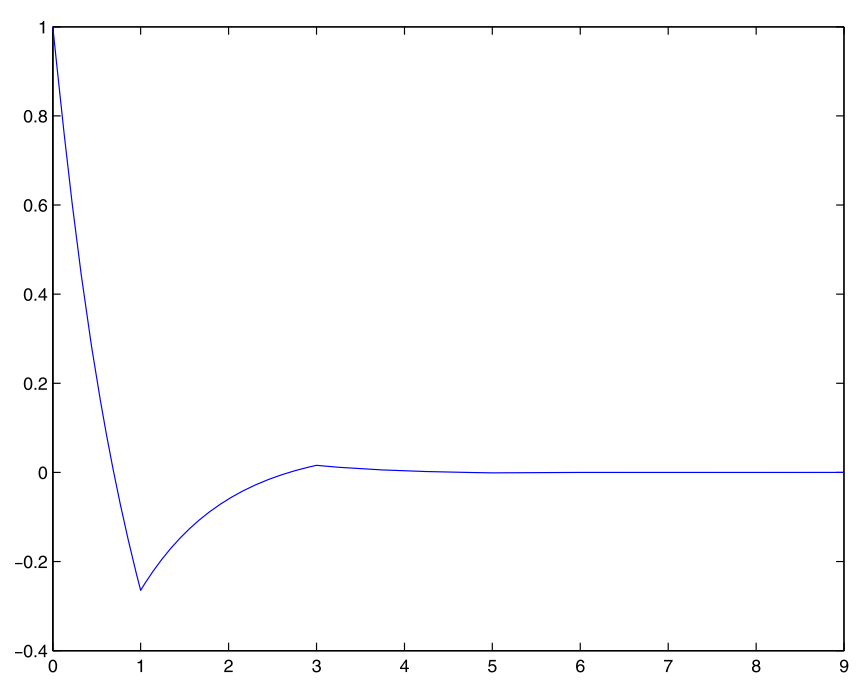

Figure 3 2-Lobatto IIIA method for (5.2).

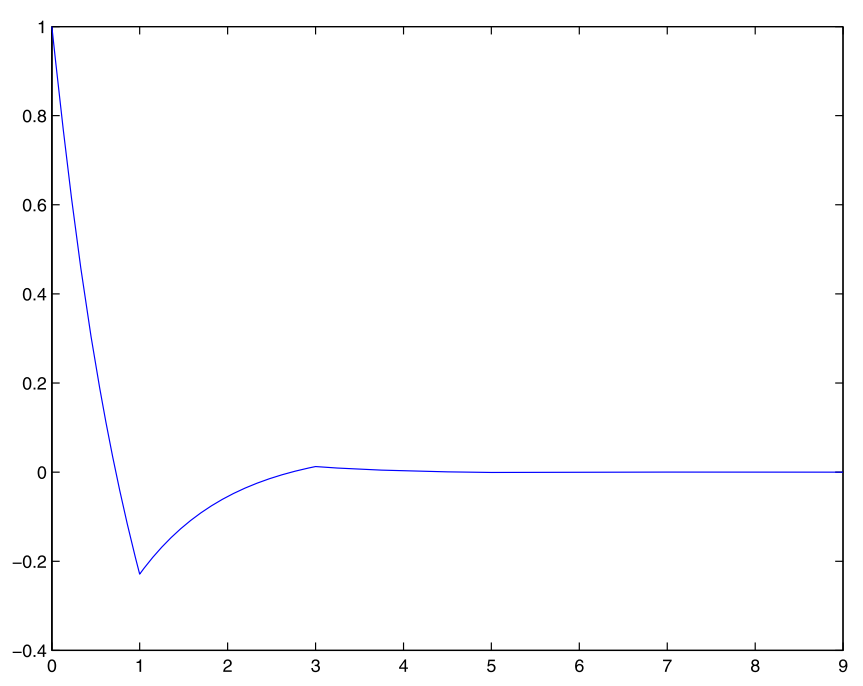

Figure 4 1-Radau IA method for (5.2).

Remark 4.12 We consider

$$
\begin{aligned}
& x^{\prime}(t)=a x(t)+a_{0} x\left(M\left[\frac{t+N}{M}\right]\right), \\
& x(0)=x_{0},
\end{aligned}
$$

where $M, N$ are positive integers and $M=2 N$.

We can obtain the same results about the stability and oscillation as Eq. (1.2), i.e.,

(i) The Runge-Kutta method preserves the asymptotic stability of Eq. (4.4) if $R(x) \leq e^{x}$.

(ii) The Runge-Kutta method preserves the oscillation of Eq. (4.4) if

$$
R(x) \geq e^{x} \quad \text { for } a>0, \quad R(x) \leq e^{x} \quad \text { for } a<0 .
$$




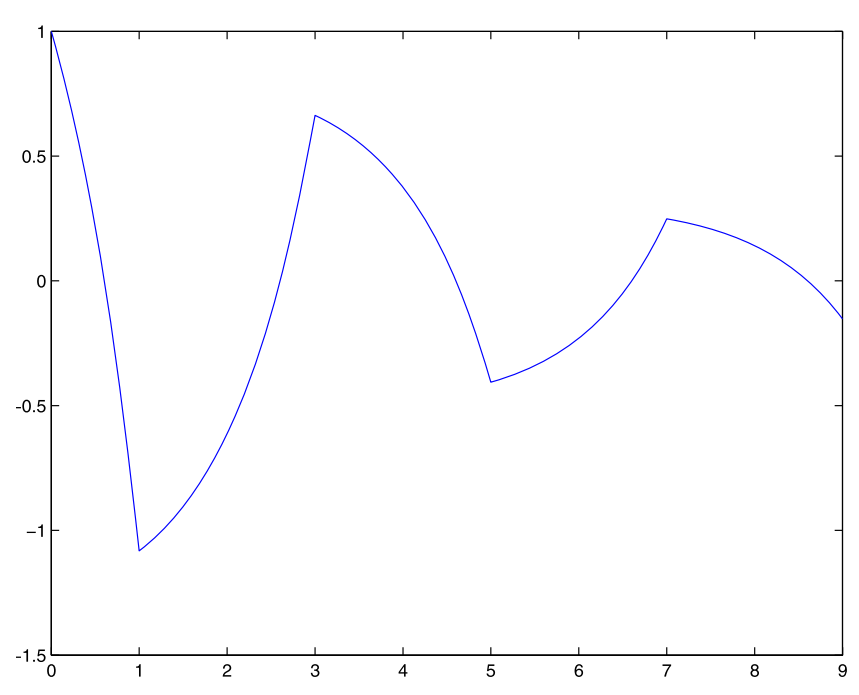

Figure 5 3-Gauss method for (5.3).

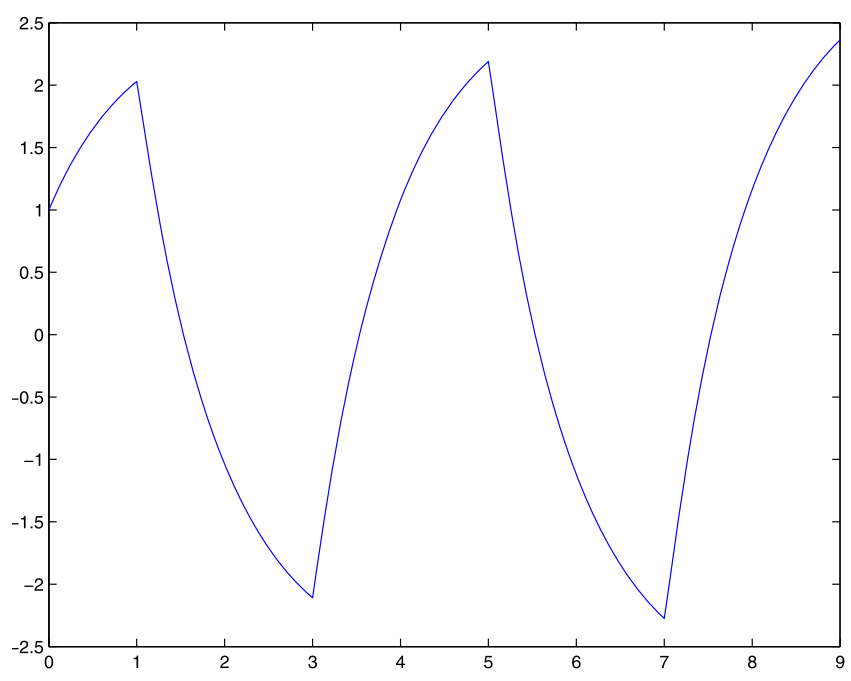

Figure 6 Explicit Euler method for (5.4).

Hence the Corollary 4.6, 4.10 and Theorem 4.7, 4.11 hold.

\section{Numerical experiments}

In this section, we give some examples to illustrate the conclusions in the paper. To illustrate the stability, we consider the following two problems:

$$
\begin{aligned}
& \left\{\begin{array}{l}
\dot{x}(t)=x(t)-1.9207 x\left(2\left[\frac{t+1}{2}\right]\right), \quad t>0, \\
x(0)=1,
\end{array}\right. \\
& \left\{\begin{array}{l}
\dot{x}(t)=-x(t)-x\left(2\left[\frac{t+1}{2}\right]\right), \quad t>0, \\
x(0)=1 .
\end{array}\right.
\end{aligned}
$$




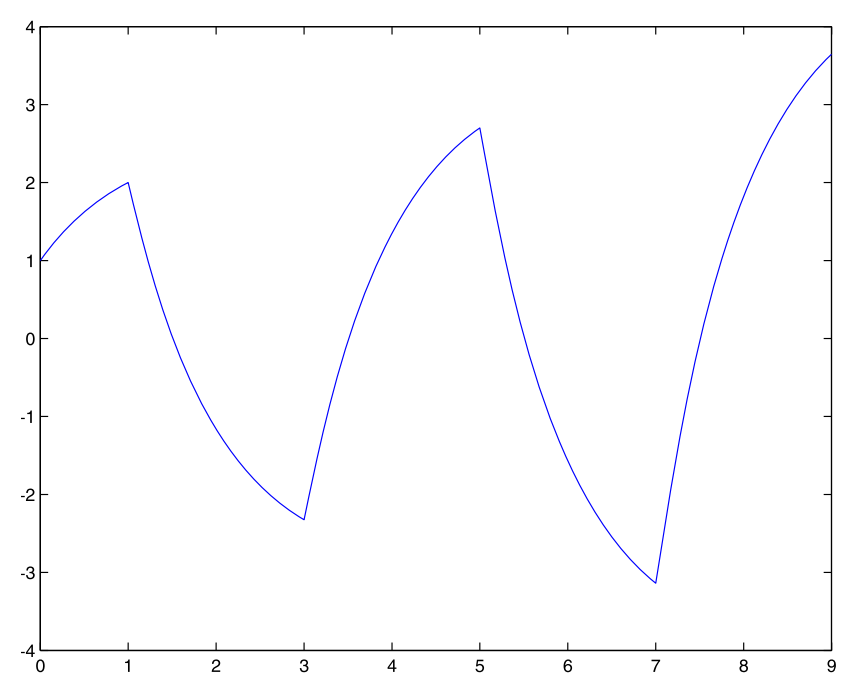

Figure 7 2-Radau IA method for (5.4).

In Figure 1 to Figure 4, we draw the numerical solutions for Eq. (5.1) and Eq. (5.2) respectively. It is easy to see that the numerical solutions are asymptotically stable.

To illustrate the oscillation, we consider the following two problems:

$$
\begin{aligned}
& \left\{\begin{array}{l}
\dot{x}(t)=x(t)-2.2117 x\left(2\left[\frac{t+1}{2}\right]\right), \quad t>0, \\
x(0)=1,
\end{array}\right. \\
& \left\{\begin{array}{l}
\dot{x}(t)=-x(t)+2.5820 x\left(2\left[\frac{t+1}{2}\right]\right), \quad t>0, \\
x(0)=1 .
\end{array}\right.
\end{aligned}
$$

In Figure 5 to Figure 7, we draw the numerical solutions for Eq. (5.3) and Eq. (5.4) respectively. It is easy to see that the numerical solutions are oscillatory.

\section{Competing interests}

The authors declare that they have no competing interests.

\section{Authors' contributions}

The authors have equal contributions to each part of this article. All the authors read and approved the final manuscript.

\section{Acknowledgements}

The financial support from the National Natural Science Foundation of China (No.11071050) is gratefully acknowledged.

Received: 25 July 2012 Accepted: 25 November 2012 Published: 10 December 2012

\section{References}

1. Cooke, KL, Wiener, J: An equation alternately of retarded and advanced type. Proc. Am. Math. Soc. 99, 726-732 (1987)

2. Jayasree, KN, Deo, SG: Variation of parameters formula for the equation of Cooke and Wiener. Proc. Am. Math. Soc $112,75-80(1991)$

3. Wiener, J, Aftabizadeh, AR: Differential equations alternately of retarded and advanced type. J. Math. Anal. Appl. 129, 243-255 (1988)

4. Busenberg, S, Cooke, KL: Models of vertically transmitted diseases with sequential-continuous dynamics. In: $\checkmark$ Lakshmikantham (Ed.) Nonlinear Phenomena in Mathematical Sciences. Academic Press, New York (1982)

5. Liu, MZ, Song, MH, Yang, ZW: Stability of Runge-Kutta methods in the numerical solution of equation $u^{\prime}(t)=a u(t)+a_{0} u([t])$. J. Comput. Appl. Math. 166, 361-370 (2004)

6. Song, MH, Yang, ZW, Liu, MZ: Stability of $\theta$-methods advanced differential equations with piecewise continuous argument. Comput. Math. Appl. 49, 1295-1301 (2005)

7. Yang, ZW, Liu, MZ, Song, MH: Stability of Runge-Kutta methods in the numerical solution of equation $u^{\prime}(t)=a u(t)+a_{0} u([t])+a_{1} u([t-1])$. Appl. Math. Comput. 162, 37-50 (2005) 
8. Liu, MZ, Gao, JF, Yang, ZW: Oscillation analysis of numerical solution in the $\theta$-methods for equation $x^{\prime}(t)+a x(t)+a_{1} x([t-1])=0$. Appl. Math. Comput. 186(1), 566-578 (2007)

9. Wiener, J: Generalized Solutions of Functional Differential Equations. World Scientific, Singapore (1993)

10. Györi, I, Ladas, G: Oscillation Theory of Delay Differential Equations. Clarendon Press, Oxford (1991)

11. Ran, XJ, Liu, MZ, Zhu, QY: Numerical methods for impulsive differential equation. Math. Comput. Model. 48(1-2), 46-55 (2007)

12. Hairer, E, Wanner, G: Solving Ordinary Differential Equations II, Stiff and Differential Algebraic Problems. Springer, New York (1993)

13. Wanner, G, Hairer, E, Nøsett, SP: Order stars and stability theorems. BIT Numer. Math. 18, 475-489 (1978)

doi:10.1186/1029-242X-2012-290

Cite this article as: Song and Liu: Numerical stability and oscillation of the Runge-Kutta methods for the differential equations with piecewise continuous arguments alternately of retarded and advanced type. Journal of Inequalities and Applications 2012 2012:290.

\section{Submit your manuscript to a SpringerOpen ${ }^{\ominus}$ journal and benefit from:}

- Convenient online submission

- Rigorous peer review

Immediate publication on acceptance

- Open access: articles freely available online

- High visibility within the field

- Retaining the copyright to your article 